TRANSACTIONS OF THE

AMERICAN MATHEMATICAL SOCIETY

Volume 353, Number 3, Pages 1089-1102

S 0002-9947(00)02641-6

Article electronically published on October 11, 2000

\title{
NEW RANGE THEOREMS FOR THE DUAL RADON TRANSFORM
}

\author{
ALEXANDER KATSEVICH
}

\begin{abstract}
Three new range theorems are established for the dual Radon transform $R^{*}$ : on $C^{\infty}$ functions that do not decay fast at infinity (and admit an asymptotic expansion), on $\mathcal{S}\left(Z_{n}\right)$, and on $C_{0}^{\infty}\left(Z_{n}\right)$. Here $Z_{n}:=S^{n-1} \times \mathbb{R}$, and $R^{*}$ acts on even functions $\mu(\alpha, p)=\mu(-\alpha,-p),(\alpha, p) \in Z_{n}$.
\end{abstract}

\section{Introduction AND StATEMEnt of the MAin RESUlts}

A number of range theorems for the Radon transform $R$ are known. A partial list includes ranges of $R$ on compactly supported $C^{\infty}$ functions, on rapidly decreasing $C^{\infty}$ functions, on compactly supported functions from Sobolev's spaces, on $C^{\infty}$ functions that do not decay rapidly at infinity, on compactly supported distributions, on distributions of rapid decay, etc. GGV66, Hel80, Hel82, Her83. Her84, Kat97, LP70, Lou84, Lud60, Ram95, SSW77. However, less is known about the dual Radon transform $R^{*}$ and how it acts on different spaces of functions. In particular, it was shown in Her84 that $R^{*}: \mathcal{D}^{\prime}\left(Z_{n}\right) \rightarrow \mathcal{D}^{\prime}\left(\mathbb{R}^{n}\right)$ is not surjective. An asymptotic expansion of $f(r \beta)=R^{*} \mu, \mu \in \mathcal{S}\left(Z_{n}\right)$, as $r \rightarrow \infty$, and an inversion formula for $R^{*}$ have been obtained in [Sol87, SM88] (see also [Hel65, Gon84, Gon87. for earlier inversion formulas and [Ram96] for a generalization to nonsmooth functions). A relationship between singular supports of $f=R^{*} \mu$ and $\mu$ is established in [Ram96. Most of the known explicit characterizations of ranges of $R^{*}$ are based on the identity $R^{*} K R f=f, f \in \mathcal{S}\left(\mathbb{R}^{n}\right)$, that is, the range of $R^{*}$ acting on the space $X=K R \mathcal{S}$ is $\mathcal{S}$ (see e.g. Lud60 Ram96, RK96]). Here $K$ is a certain singular operator (see (1.4) below). Even though the space $X$ can be conveniently described by moment conditions, it is of interest to find ranges of $R^{*}$ on conventional spaces of functions, that is, on such spaces where one does not assume any moment conditions, and imposes only some natural conditions on the rate of decay at infinity or compactness of support. Three such results are obtained in this paper. We establish range theorems for $R^{*}$ on $C^{\infty}$ functions that do not decay fast at infinity (and admit an asymptotic expansion), on $\mathcal{S}\left(Z_{n}\right)$, and on $C_{0}^{\infty}\left(Z_{n}\right)$. Also, we give a simple formula that relates the coefficients of the expansion of $f=R^{*} \mu$ and moments of $\mu$. Even though such a relationship is known (see (3.5) in [Sol87]), the formula given here is shorter and more convenient to work with. For example, our derivation of the range of $R^{*}$ on $\mathcal{S}$ relies heavily on this formula.

Received by the editors January 20, 1998 and, in revised form, June 24, 1999.

2000 Mathematics Subject Classification. Primary 44A12.

Key words and phrases. Dual Radon transform, range theorems, asymptotic expansions.

This research was supported in part by NSF grant DMS-9704285. 
The following notation is used throughout the paper. The Radon transform $R$ and its dual $R^{*}$ are defined by

$$
R f(\alpha, p)=\int_{\mathbb{R}^{n}} f(x) \delta(\alpha \cdot x-p) d x, R^{*} \mu(x)=\int_{S^{n-1}} \mu(\alpha, \alpha \cdot x) d \alpha,
$$

where $S^{n-1}$ is the unit sphere in $\mathbb{R}^{n}$. Since $R^{*}$ annihilates odd functions, we consider $R^{*}$ acting only on even functions: $\mu(\alpha, p)=\mu(-\alpha,-p)$. The inversion formula for $R^{*}$ is given by (see (8.2) in [Sol87])

$$
\gamma_{n} R \mathcal{I}^{1-n} R^{*} \mu=\mu, \mu \in \mathcal{S}\left(Z_{n}\right), \gamma_{n}:=\frac{1}{2(2 \pi)^{n-1}}, Z_{n}:=S^{n-1} \times \mathbb{R}
$$

where the operator $\mathcal{I}^{a}$ (the Riesz potential) is defined by

$$
\mathcal{I}^{a} f:=\mathcal{F}^{-1}\left(|\xi|^{-a} \mathcal{F} f\right),
$$

and $\mathcal{F}$ is the $n$-dimensional Fourier transform. Similarly, $F$ denotes the onedimensional Fourier transform acting with respect to the second variable. The operator $K$ referred to above is

$$
K \mu:=\gamma_{n} F^{-1}\left(|t|^{n-1} F \mu\right), \mu \in \mathcal{S}\left(Z_{n}\right) .
$$

$\mathcal{S}\left(Z_{n}\right)$ and $\mathcal{S}\left(\mathbb{R}^{n}\right)$ are the Schwartz spaces of functions that decrease rapidly with all their derivatives, and $Y_{m}$ denotes an arbitrary spherical harmonics of degree $m$.

First, we will state the main results. The proofs are given in the following two sections.

Theorem 1. Let $\mathcal{X}$ be the space of $C^{\infty}\left(Z_{n}\right)$ even functions that admit the expansion

$$
\mu(\alpha, p) \sim \sum_{k=0}^{\infty} \sum_{m=0}^{M_{k}} \mu_{k, m}(\alpha) \frac{\ln ^{m} p}{p^{k+1}}, p \rightarrow+\infty, \mu_{k, m} \in C^{\infty}\left(S^{n-1}\right),
$$

which is uniform with respect to $\alpha \in S^{n-1}$ and can be differentiated with respect to $\alpha$ and $p$ any number of times. Let $\mathcal{Y}$ be the space of $C^{\infty}\left(\mathbb{R}^{n}\right)$ functions that admit the expansion

$$
f(r \beta) \sim \sum_{k=0}^{\infty} \sum_{j=0}^{J_{k}} f_{k, j}(\beta) \frac{\ln ^{j} r}{r^{k+1}}, r \rightarrow+\infty, f_{k, j} \in C^{\infty}\left(S^{n-1}\right),
$$

which is uniform with respect to $\beta \in S^{n-1}$ and can be differentiated with respect to $x=r \beta$ any number of times. Then $R^{*} \mathcal{X}=\mathcal{Y}, R^{*}: \mathcal{X} \rightarrow \mathcal{Y}$ is injective, and the inversion formula $\left(R^{*}\right)^{-1}=\gamma_{n} R \mathcal{I}^{1-n}$ holds on $\mathcal{Y}$.

Theorem 2. Let $\mathcal{Y}_{1} \subset \mathcal{Y}$ be the space of $C^{\infty}\left(\mathbb{R}^{n}\right)$ functions that admit the expansion

$$
\begin{aligned}
& f(r \beta) \sim \sum_{k=0}^{\infty} \frac{f_{k}(\beta)}{r^{k+1}}, r \rightarrow+\infty, \\
& f_{k} \in C^{\infty}\left(S^{n-1}\right), f_{k}(-\beta)=(-1)^{k} f_{k}(\beta),
\end{aligned}
$$

which is uniform with respect to $\beta \in S^{n-1}$ and can be differentiated with respect to $x=r \beta$ any number of times. Suppose, in addition, that

$$
\int_{S^{n-1}} f_{k}(\beta) Y_{m}(\beta) d \beta=0, m \leq k-(n-1), \quad \text { if } k \geq n-1 \text { and } n \text { is odd }
$$


and, for any polynomial $P$, the finite part of the limit

$$
M_{P}=F . p . \lim _{\epsilon \rightarrow 0^{+}} \int_{\mathbb{R}^{n}} f(x) P(x) e^{-\epsilon|x|} d x=0, \quad \text { if } n \text { is even. }
$$

Then $R^{*} \mathcal{S}\left(Z_{n}\right)=\mathcal{Y}_{1}$. Let $\mathcal{Y}_{2} \subset \mathcal{Y}_{1}$ be the set of functions such that given any spherical harmonics $Y_{m}$, one has

$$
\int_{S^{n-1}} f(r \beta) Y_{m}(\beta) d \beta=\sum_{k=0}^{\infty} r^{-(k+1)} \int_{S^{n-1}} f_{k}(\beta) Y_{m}(\beta) d \beta, r>A,
$$

where $A>0$ is some constant independent of $m$ (A, of course, depends on $f)$. Then $R^{*} C_{0}^{\infty}\left(Z_{n}\right)=\mathcal{Y}_{2}$. Moreover, if $f \in \mathcal{Y}_{2}$ and $A>0$ are such that (1.10) holds, then $\mu(\alpha, p)=\left(R^{*}\right)^{-1} f=0,|p|>A$.

Proposition 1. Let $f=R^{*} \mu$ for some $\mu \in \mathcal{S}\left(Z_{n}\right)$. Then the coefficient $f_{k}(\beta)$ in the expansion of $f$ can be expressed in terms of $\mu$ as follows:

$$
f_{k}(\beta)=\frac{(-1)^{k}}{k !} \int_{S^{n-1}} \int_{-\infty}^{\infty} \delta^{(k)}(\alpha \cdot \beta) p^{k} \mu(\alpha, p) d p d \alpha
$$

or, equivalently,

$$
f_{k}(\beta)=\frac{1}{k !} \int_{\substack{\omega \cdot \beta=0 \\|\omega|=1}} \int_{-\infty}^{\infty} p^{k}\left(\frac{\partial}{\partial t}\right)^{k}\left[\mu\left(t \beta+\sqrt{1-t^{2}} \omega, p\right)\left(1-t^{2}\right)^{\frac{n-3}{2}}\right]_{t=0} d p d \omega
$$

\section{Proof of Theorem 1}

First, two auxiliary lemmas will be stated and proved, and then the proof of Theorem 1 will be given.

Lemma 1. Consider the function

$$
f(\lambda)=\int_{0}^{\infty} \int_{-1}^{1} g(x, y) e^{i \lambda x y} d y d x
$$

where $g(x, y)$ is $C^{\infty}((0, \infty))$ and $C_{0}^{\infty}([-1,1])$ with respect to $x$ and $y$, respectively, $g$ and all of its derivatives decay faster than any power of $1 / x$ as $x \rightarrow \infty$, and

$$
g(x, y) \sim \sum_{k=0}^{\infty} \sum_{m=0}^{M_{k}} g_{k, m}(y) x^{k} \ln ^{m} x, x \rightarrow 0^{+}, g_{k, m} \in C_{0}^{\infty}([-1,1])
$$

the expansion is uniform with respect to $y \in[-1,1]$, and can be differentiated with respect to $x>0$ and $y \in[-1,1]$. Then

$$
f(\lambda) \sim \sum_{k=0}^{\infty} \sum_{m=0}^{M_{k}} \int_{-1}^{1} \Psi_{k, m}(\lambda y) g_{k, m}(y) d y, \lambda \rightarrow \infty
$$

where $\Psi_{k, m}$ are defined via the one-dimensional Fourier transform

$$
\Psi_{k, m}(t):=F_{s \rightarrow t}\left(s_{+}^{k} \ln ^{m} s_{+}\right),
$$

and the expansion can be differentiated with respect to $\lambda$. 
Changing variables in the Fourier transform integral which defines $\Psi_{k, m}$, it is easy to see that

$$
\Psi_{k, m}(\lambda y)=\lambda^{-(k+1)} \sum_{j=0}^{m}\left(\begin{array}{c}
m \\
j
\end{array}\right)(-\ln \lambda)^{j} \Psi_{k, m-j}(y) .
$$

Therefore, (2.3) is a bona fide asymptotic expansion. We used the form (2.3) rather than the standard explicit form because the former is more compact and convenient for our purposes.

Even though Lemma 1 appears to be a standard result, the author failed to find a reference where it was stated in the same form as above. The closest result is given in [Fed77]. However, the book is not readily available and, moreover, the corresponding lemma in [Fed77] is stated without logarithmic terms. Since Lemma 1 is central to the proof of the main results, a short derivation of the asymptotic expansion in (2.3) is presented here for convenience of the reader.

Proof. Substituting (2.2) into (2.1), we find formally

$$
\begin{aligned}
f(\lambda) & \sim \sum_{k=0}^{\infty} \sum_{m=0}^{M_{k}} \int_{0}^{\infty} \int_{-\infty}^{\infty} g_{k, m}(y) x^{k} \ln ^{m} x e^{i \lambda x y} d y d x \\
& =\sum_{k=0}^{\infty} \sum_{m=0}^{M_{k}} \int_{-\infty}^{\infty} g_{k, m}(y) F_{x \rightarrow \lambda y}\left(x_{+}^{k} \ln ^{m} x_{+}\right) d y
\end{aligned}
$$

Let us prove that the formal expansion in (2.6) is asymptotic. Define the function $I_{K}(\lambda)$ as follows:

$$
\begin{aligned}
& I_{K}(\lambda):=\int_{0}^{\infty} \int_{-\infty}^{\infty} g_{K}(x, y) e^{i \lambda x y} d y d x \\
& g_{K}(x, y):=g(x, y)-\sum_{k=0}^{K-1} \sum_{m=0}^{M_{k}} g_{k, m}(y) x^{k} \ln ^{m} x .
\end{aligned}
$$

We have to show that the larger $K$ is the faster $I_{K}(\lambda)$ decreases as $\lambda \rightarrow \infty$. Fix any $\varphi \in C^{\infty}\left(\mathbb{R}_{+}\right)$such that $\varphi(x)=1, x<1$, and $\varphi(x)=0, x>2$. Then the same integral as in (2.7), except that the integrand is multiplied by $(1-\varphi(x))$, is $O\left(\lambda^{-\infty}\right), \lambda \rightarrow \infty$, because we can integrate by parts with respect to $y$, any number of times and $x$ is bounded away from zero on $\operatorname{supp}(1-\varphi)$. Since $\varphi(x) g_{K}(x, y)$ is compactly supported and $g_{K}(x, y)=O\left(x^{K} \ln ^{M_{K}} x\right), x \rightarrow 0^{+}$, we have

$$
\begin{aligned}
I_{K}(\lambda) & =\int_{0}^{\infty} \int_{-\infty}^{\infty} \varphi(x) g_{K}(x, y) e^{i \lambda x y} d y d x \\
& =\left(\frac{i}{\lambda}\right)^{K} \int_{0}^{\infty} \int_{-\infty}^{\infty} \frac{(\partial / \partial y)^{K}\left(\varphi(x) g_{K}(x, y)\right)}{x^{K}} e^{i \lambda x y} d y d x,
\end{aligned}
$$

and the last integral is absolutely convergent uniformly with respect to $\lambda \in \mathbb{R}_{+}$. Therefore, $I(\lambda)$ decays at least as fast as $O\left(1 / \lambda^{K}\right), \lambda \rightarrow \infty$, which proves the desired result. Differentiating (2.8) with respect to $\lambda$ under the integral sign and then integrating by parts with respect to $y$ one more time (this is possible because after the differentiation an additional factor $x$ appears), we establish differentiability of (2.6) with respect to $\lambda$. 
Lemma 2. Pick any function $g \in C^{\infty}\left(\mathbb{R}^{n}\right)$ such that

$$
g(r \beta) \sim \sum_{k=0}^{\infty} \sum_{j=0}^{J_{k}} g_{k, j}(\beta) \frac{\ln ^{j} r}{r^{k+n}}, r \rightarrow \infty, g_{k, j} \in C^{\infty}\left(S^{n-1}\right),
$$

the expansion is uniform with respect to $\beta \in S^{n-1}$ and can be differentiated with respect to $x=r \beta$. Then $R g \in C^{\infty}\left(Z_{n}\right)$,

$$
R g(\alpha, p) \sim \sum_{k=0}^{\infty} \sum_{j=0}^{J_{k}} p^{-(k+1)} \int_{\alpha \cdot \beta>0}(\alpha \cdot \beta)^{k} \ln ^{j}(p /(\alpha \cdot \beta)) g_{k, j}(\beta) d \beta, p \rightarrow+\infty,
$$

the expansion is uniform with respect to $\alpha \in S^{n-1}$ and can be differentiated with respect to $\alpha$ and $p$.

Proof. Clearly, $g(r \beta)$ is absolutely integrable over any hyperplane in $\mathbb{R}^{n}$, therefore $R g(\alpha, p)$ exists for all $(\alpha, p) \in Z_{n}$. Consider a subset $\Omega_{\epsilon}$ of $S^{n-1}$ defined, for example, by the conditions $|\hat{\alpha}|<\epsilon, \alpha_{n}>0$, where $\alpha=\left(\hat{\alpha}, \alpha_{n}\right), \hat{\alpha}=\left(\alpha_{1}, \ldots, \alpha_{n-1}\right)$. Suffice it to show that $R g(\alpha, p)$ is differentiable for $\alpha \in \Omega_{\epsilon}$. Since $\alpha_{n}=\sqrt{1-|\hat{\alpha}|^{2}} \neq$ 0 if $\alpha \in \Omega_{\epsilon}$, we have

$$
R g(\alpha, p)=\frac{1}{\alpha_{n}} \int_{\mathbb{R}^{n-1}} g\left(\hat{x},(p-\hat{\alpha} \cdot \hat{x}) / \alpha_{n}\right) d \hat{x}, \alpha \in \Omega_{\epsilon},
$$

and, as was mentioned, (2.9) implies that the integral is absolutely convergent. Differentiating on both sides of (2.11) we get, after simple transformations,

$$
\begin{array}{r}
\frac{\partial}{\partial \alpha_{j}} R g(\alpha, p)=\frac{1}{\alpha_{n}^{2}}\left\{\alpha_{j} R\left(g+x_{n}\left(\partial g / \partial x_{n}\right)\right)(\alpha, p)-\alpha_{n} R\left(x_{j}\left(\partial g / \partial x_{n}\right)\right)(\alpha, p)\right\}, \\
\alpha \in \Omega_{\epsilon}, 1 \leq j \leq n-1 .
\end{array}
$$

Since expansion (2.9) can be differentiated with respect to $x=r \beta$, we see that $g+x_{n}\left(\partial g / \partial x_{n}\right)=O\left(\ln ^{J_{0}}|x| /|x|^{n}\right)$ and $x_{j}\left(\partial g / \partial x_{n}\right)=O\left(\ln ^{J_{0}}|x| /|x|^{n}\right)$ as $|x| \rightarrow$ $\infty$. Therefore, the integrals that define the Radon transform of the two functions are absolutely convergent and differentiation under the integral sign is justified. Repeating the above argument for derivatives of higher order we show that $R g(\alpha, p)$ is differentiable with respect to $\alpha$. Since

$$
\frac{\partial}{\partial p} R g(\alpha, p)=\frac{1}{\alpha_{n}} R\left(\partial g / \partial x_{n}\right)(\alpha, p), \alpha \in \Omega_{\epsilon},
$$

we see that $R g(\alpha, p)$ is differentiable with respect to $p$.

Let $R_{k j}(r)$ be any smooth function such that $R_{k j}(r)=\ln ^{j} r / r^{k+n}, r>1$. Then, for $p>1$ and any $g_{k j} \in C^{\infty}\left(S^{n-1}\right)$,

$$
\begin{aligned}
R\left(g_{k j}(\beta) R_{k j}(r)\right)(\alpha, p) & =\int_{S^{n-1}} g_{k, j}(\beta) \int_{0}^{\infty} R_{k j}(r) \delta(r \alpha \cdot \beta-p) r^{n-1} d r d \beta \\
& =\int_{\alpha \cdot \beta>0} g_{k, j}(\beta) \int_{p}^{\infty} \frac{\ln ^{j} r}{r^{k+1}} \delta(r \alpha \cdot \beta-p) d r d \beta \\
& =\frac{1}{p^{k+1}} \int_{\alpha \cdot \beta>0}(\alpha \cdot \beta)^{k} \ln ^{j}(p /(\alpha \cdot \beta)) g_{k, j}(\beta) d \beta,
\end{aligned}
$$


which immediately implies the formal expansion (2.10). The asymptotic nature of the expansion follows from the fact that, if $g(x)=o\left(|x|^{-(n+k)}\right),|x| \rightarrow \infty, k \geq 0$, then $R g(\alpha, p)=o\left(p^{-(k+1)}\right), p \rightarrow \infty$. This also implies that the expansion is uniform with respect to $\alpha \in S^{n-1}$.

Let us show that (2.10) can be differentiated with respect to $\alpha$ and $p$. By what was already proved, the functions $R\left(g+x_{n}\left(\partial g / \partial x_{n}\right)\right)$ and $R\left(x_{j}\left(\partial g / \partial x_{n}\right)\right)$ admit uniform expansion of the type (2.10). Equation (2.12) implies that derivatives of $R g(\alpha, p)$ with respect to $\alpha$ admit uniform expansion analogous to (2.10). Therefore, (2.10) can be differentiated with respect to $\alpha$. In (2.10) the asymptotic sequence is $\left\{\varphi_{j, k}(p):=\ln ^{j} p / p^{k+1}\right\}, 0 \leq j \leq J_{k}, k \geq 0$. Clearly, the sequence consisting of derivatives of $\varphi_{j, k}:\left\{(d / d p)^{l} \varphi_{j, k}(p)\right\}$, where $l$ is the same for all $j$ and $k$, is still asymptotic. Hence (2.13), differentiability of (2.9), and what we have already proved, imply that $\partial^{l}(R g) / \partial p^{l}$ admits an expansion with respect to the sequence $\left\{\varphi_{j, k}^{(l)}\right\}$. Therefore, (2.10) can be differentiated with respect to $p$.

Proof of Theorem 11. Let us check the inclusion $R^{*} \mathcal{X} \subset \mathcal{Y}$. Given any $\mu(\alpha, p) \in \mathcal{X}$ we establish that $F \mu(\alpha, \lambda)=F_{p \rightarrow \lambda} \mu(\alpha, p)$ is integrable at $\lambda=0, F \mu(\alpha, \lambda) \in$ $C^{\infty}\left(S^{n-1} \times(\mathbb{R} \backslash 0)\right)$, and

$$
\left|\frac{\partial^{\gamma}}{\partial \alpha^{\gamma}} \frac{\partial^{l}}{\partial \lambda^{l}} F \mu(\alpha, \lambda)\right| \leq c_{\gamma l N}(1+|\lambda|)^{-N},|\lambda|>1, \alpha \in S^{n-1},
$$

for any multi-index $\gamma$, integers $l, N=0,1,2, \ldots$, and some $c_{\gamma l N}>0$. Moreover,

$$
F \mu(\alpha, \lambda) \sim \sum_{k=0}^{\infty} \sum_{m=0}^{M_{k}+1} \tilde{\mu}_{k, m}(\alpha) \lambda^{k} \ln ^{m} \lambda, \lambda \rightarrow 0^{+}, \tilde{\mu}_{k, m} \in C^{\infty}\left(S^{n-1}\right)
$$

the expansion is uniform with respect to $\alpha \in S^{n-1}$ and can be differentiated with respect to $\alpha$ and $\lambda$. Property (2.15) follows from the assumption that 1.5 can be differentiated with respect to $\alpha, p$, and the obvious identity

$$
\frac{\partial^{\gamma}}{\partial \alpha^{\gamma}} \frac{\partial^{l}}{\partial \lambda^{l}} F \mu(\alpha, \lambda)=\frac{\partial^{\gamma}}{\partial \alpha^{\gamma}} \frac{\partial^{l}}{\partial \lambda^{l}}\left\{\frac{1}{(-i \lambda)^{j}} \int_{-\infty}^{\infty} \frac{\partial^{j} \mu(\alpha, p)}{\partial p^{j}} e^{i \lambda p} d p\right\}
$$

where $j>0$ can be taken arbitrarily large. Expansion 2.16) and integrability of $F \mu(\alpha, \lambda)$ at $\lambda=0$ follow if we apply the results of [BH86], pp. 206 and 207, and the results of [GS64] on regularization of distributions to the integrals on the right-hand side of the equation

$$
F \mu(\alpha, \lambda)=\int_{-\infty}^{\infty} \mu(\alpha, p) e^{i \lambda p} d p=\int_{0}^{\infty} \mu(\alpha, p) e^{i \lambda p} d p+\int_{0}^{\infty} \mu(-\alpha, p) e^{-i \lambda p} d p
$$

Expansion (2.16) is uniform because (1.5) is uniform, and differentiability of (2.16) is obvious. The number of logarithmic terms in (2.16) is one plus the number of the corresponding terms in the expansion of $\mu$ because the poles of the (analytically continued) Mellin transforms of $e^{i t}$ and $\mu(\alpha, p)$ (with respect to $p$ ) interfere with each other. 
Using that $\mu$ is even, one has

$$
\begin{aligned}
R^{*} \mu(r \beta) & =\int_{S^{n-1}} \mu(\alpha, r \alpha \cdot \beta) d \alpha \\
& =\frac{1}{\pi} \int_{0}^{\infty} \int_{S^{n-1}} F \mu(\alpha, \lambda) e^{-i \lambda r \alpha \cdot \beta} d \alpha d \lambda \\
& =\int_{0}^{\infty} \int_{-1}^{1}\left(1-t^{2}\right)^{(n-3) / 2} \tilde{\mu}_{\beta}(\lambda, t) e^{-i \lambda r t} d t d \lambda
\end{aligned}
$$

where

$$
\tilde{\mu}_{\beta}(\lambda, t):=\frac{1}{\pi} \int_{w \in S_{\beta \perp}^{n-2}} F \mu\left(t \beta+\sqrt{1-t^{2}} w, \lambda\right) d w .
$$

Here $S_{\beta^{\perp}}^{n-2}$ is the unit sphere in the hyperplane passing through the origin perpendicular to $\beta$. Substitution of (2.16) into (2.20) implies that $\tilde{\mu}_{\beta}(\lambda, t)$ admits an expansion

$$
\begin{aligned}
& \tilde{\mu}_{\beta}(\lambda, t) \sim \sum_{k=0}^{\infty} \sum_{m=0}^{M_{k}+1} \check{\mu}_{k, m}(t, \beta) \lambda^{k} \ln ^{m} \lambda, \lambda \rightarrow 0^{+}, \\
& \check{\mu}_{k, m}(t, \beta)=\frac{1}{\pi} \int_{w \in S_{\beta}^{n-2}} \tilde{\mu}_{k, m}\left(t \beta+\sqrt{1-t^{2}} w\right) d w .
\end{aligned}
$$

$\check{\mu}_{k, m}$ is $C^{\infty}((-1,1)) \cap C([-1,1])$ and $C^{\infty}\left(S^{n-1}\right)$ with respect to $t$ and $\beta$, respectively, the expansion is uniform with respect to $(t, \beta) \in[-1,1] \times S^{n-1}$ and can be differentiated with respect to $(t, \beta)$ belonging to compact subsets of $(-1,1) \times S^{n-1}$.

Pick any $\eta(y) \in C_{0}^{\infty}([-1,1])$ such that $\eta(y) \equiv 1,|y|<1 / 2$. We have $R^{*} \mu(r \beta)=$ $f_{1}(r, \beta)+f_{2}(r, \beta)$, where

$$
\begin{aligned}
& f_{1}(r, \beta):=\int_{1 / 2 \leq|t| \leq 1}(1-\eta(t))\left(1-t^{2}\right)^{(n-3) / 2} \int_{0}^{\infty} \tilde{\mu}_{\beta}(\lambda, t) e^{-i \lambda r t} d \lambda d t, \\
& f_{2}(r, \beta):=\int_{0}^{\infty} \int_{-\infty}^{\infty} \eta(t)\left(1-t^{2}\right)^{(n-3) / 2} \tilde{\mu}_{\beta}(\lambda, t) e^{-i \lambda r t} d t d \lambda .
\end{aligned}
$$

The asymptotics of $f_{1}$ can be found using well-known one-dimensional methods (see e.g. [BH86, Won89]). We have

$$
\int_{0}^{\infty} \tilde{\mu}_{\beta}(\lambda, t) e^{-i \lambda s} d \lambda \sim \sum_{k=0}^{\infty} \sum_{m=0}^{M_{k}+1} \check{\mu}_{k, m}(t, \beta) \overline{\Psi_{k, m}(s)}, s \rightarrow \infty,
$$

where $\Psi_{k, m}$ are defined by (2.4) and the overbar denotes complex conjugation. Since $1 / 2 \leq|t| \leq 1$ in (2.22), letting $s=r t$ in (2.24), substituting into (2.22), using (2.5), and integrating with respect to $t$, it is elementary to see that $f_{1}$ admits an expansion of the form (1.6). Applying Lemma 1 to the function $f_{2}$, we prove that $f_{2}$ admits an expansion of the form (1.6). Since the expansion in (2.21) is uniform and differentiable with respect to $\beta \in S^{n-1}$, it is easily seen that the expansions of $f_{1}$ and $f_{2}$ are uniform and differentiable with respect to $\beta \in S^{n-1}$. This completes the proof of the inclusion $R^{*} \mathcal{X} \subset \mathcal{Y}$.

Let us now show that $\mathcal{Y} \subset R^{*} \mathcal{X}$. Fix $f \in \mathcal{Y}$. Let $n$ be odd. Denote

$$
g(x):=(-\Delta)^{(n-1) / 2} f(x), \mu:=\gamma_{n} R g .
$$


Since (1.6) is differentiable, $g$ admits an expansion of the type (2.9). Appealing to Lemma 2 we conclude that $\mu \in \mathcal{X}$. Let $n$ be even, and define $\mu$ by the equations

$$
g_{l}(x):=\left(\partial / \partial x_{l}\right)^{n-1} f(x), \alpha_{l}^{n-1} \mu:=\gamma_{n}(-1)^{n / 2} \mathcal{H} R g_{l},
$$

where $\mathcal{H}$ is the Hilbert transform with respect to $p$. Appealing to the Fourier slice theorem [Sol87], p. 329, which holds for $g_{l}$ (because $g_{l} \in L^{p}\left(\mathbb{R}^{n}\right)$ for some $p$, $1<p<n /(n-1))$, we see that $\mu$ is independent of $l$ and, moreover, satisfies

$$
F \mu(\alpha, \lambda)=\gamma_{n} \operatorname{sgn} \lambda \cdot \lambda^{n-1} \mathcal{F} f(\lambda \alpha), \lambda \neq 0, \alpha \in S^{n-1} .
$$

By Lemma [2, $R g_{l} \in \mathcal{X}$. Computing the Fourier transform, it is easily seen that $F_{p \rightarrow \lambda} R g_{l}(\alpha, p)$ admits an expansion analogous to (2.16). Multiplying by $\operatorname{sgn} \lambda$, taking the inverse Fourier transform $F_{\lambda \rightarrow p}^{-1}$, using the results of [BH86], p. 231, and taking into account that $\mu$ is independent of $l$, we prove the inclusion $\mu \in \mathcal{X}$.

It remains to check that $R^{*} \mu=f$. Using (2.27) (if $n$ is even) and (2.25) together with the Fourier slice theorem [Sol87], p. 329, applied to $g_{l}$ (if $n$ is odd), we conclude that in both cases

$$
F \mu(\alpha, \lambda):=\gamma_{n} \lambda^{n-1} \mathcal{F} f(\lambda \alpha), \lambda>0, \alpha \in S^{n-1} .
$$

In view of an obvious string of identities,

$$
\begin{aligned}
\int_{S^{n-1}} & \mu(\alpha, \alpha \cdot x) d \alpha \\
& =\frac{1}{\pi} \int_{S^{n-1}} \int_{0}^{\infty} F \mu(\alpha, \lambda) e^{-i \lambda \alpha \cdot x} d \lambda d \alpha(F \mu \text { is even }) \\
& =\frac{1}{(2 \pi)^{n}} \int_{S^{n-1}} \int_{0}^{\infty} \lambda^{n-1} \mathcal{F} f(\lambda \alpha) e^{-i \lambda \alpha \cdot x} d \lambda d \alpha(\text { by }(\underline{2.28})) \\
& =f(x) \text { (in the sense of distributions) }
\end{aligned}
$$

we conclude that $R^{*} \mu=f$ in the sense of distributions. Since both functions are $C^{\infty}$, they are equal everywhere. Equations (2.28), (2.29), and the Fourier slice theorem imply also that the inversion formula $\left(R^{*}\right)^{-1}=\gamma_{n} R \mathcal{I}^{1-n}$ holds on $\mathcal{Y}$. Estimates (2.15) and expansion (2.16) imply that $F \mu(\alpha, \lambda) \lambda^{1-n} \in L^{1}\left(\mathbb{R}^{n}\right)$, where $\lambda=|x|, \alpha=x /|x|$. Together with the second equality in 2.19) this implies injectivity of $R^{*}$.

\section{Proof of Theorem 2 and Proposition 1}

Let $\mu \in \mathcal{S}\left(Z_{n}\right)$. Then $F \mu(\alpha, \lambda) \sim \sum_{k=0}^{\infty} \tilde{\mu}_{k}(\alpha) \lambda^{k}, \lambda \rightarrow 0$. Equations (2.3), (2.4), 2.19) 2.24), and entry 22 in [GS64], p. 360, imply that $f=R^{*} \mu$ admits an expansion:

$$
\begin{aligned}
& f(r \beta) \sim \sum_{k=0}^{\infty} \frac{f_{k}(\beta)}{r^{k+1}}, f_{k}(\beta)=\frac{1}{\pi} \int_{S^{n-1}} \overline{\Psi_{k}(\alpha \cdot \beta)} \tilde{\mu}_{k}(\alpha) d \alpha, \\
& \Psi_{k}(t):=F_{s \rightarrow t}\left(s_{+}^{k}\right)=\frac{i^{k+1} k !}{t^{k+1}}+(-i)^{k} \pi \delta^{(k)}(t),
\end{aligned}
$$

where the overbar denotes complex conjugation. Since $\mu$ is even, $F \mu$ is also even and $\tilde{\mu}_{k}(-\alpha)=(-1)^{k} \tilde{\mu}_{k}(\alpha)$. Equation (3.1) implies that

$$
f_{k}(-\beta)=(-1)^{k} f_{k}(\beta) \text {. }
$$


Moreover,

$$
\int_{S^{n-1}} \frac{1}{(\alpha \cdot \beta)^{k+1}} \tilde{\mu}_{k}(\alpha) d \alpha=0 .
$$

Using (3.1) and (3.3) one immediately proves (1.11). By the Funk-Hecke theorem (cf. [RK96], p. 18), if $\tilde{\mu}_{k}(\alpha)$ contains a component $Y_{m}(\alpha)$, then $f_{k}(\beta)$ will contain the same component, but it will be multiplied by the coefficient

$$
c_{k m}=\frac{1}{\pi} \int_{S^{n-1}} \int_{S^{n-1}} Y_{m}(\alpha) Y_{m}(\beta) \overline{\Psi_{k}(\alpha \cdot \beta)} d \alpha d \beta .
$$

Substituting $\Psi_{k}(t)=\int_{0}^{\infty} s^{k} e^{i t s} d s$ and using (14.4.48) in RK96, (6.561.14) in GR94, we find

$$
\begin{aligned}
c_{k m} & =\frac{1}{\pi}(2 \pi)^{n / 2}(-i)^{m} 2^{k-0.5(n-2)} \frac{\Gamma(0.5(m+k+1))}{\Gamma(0.5(m-k+n-1))} \\
& =0, \quad \text { if } m=k-(n-1), k-(n-1)-2, \ldots
\end{aligned}
$$

Therefore, if $k \geq n-1$,

$$
\int_{S^{n-1}} f_{k}(\alpha) Y_{m}(\alpha) d \alpha=0, m=k-(n-1), k-(n-1)-2, \ldots
$$

This condition is important only when $n$ is odd, because it is automatically satisfied when $n$ is even (cf. (3.2)). This proves that $R^{*} \mathcal{S}\left(Z_{n}\right) \subset \mathcal{Y}_{1}$ if $n$ is odd. Let us now look at the generalized moments

$$
M_{P}=F \cdot p \cdot \lim _{\epsilon \rightarrow 0^{+}} \int_{\mathbb{R}^{n}} f(x) P(x) e^{-\epsilon|x|} d x,
$$

where $P$ is a polynomial and F.p. denotes the finite part. Since any polynomial can be expressed as a linear combination of 'elementary' polynomials $r^{m+2 l} Y_{m}(\beta)$, we may take $P$ of the form $P(r \beta)=r^{m+2 l} Y_{m}(\beta)$. Substitution into 3.7) yields (cf. (6.623.2) in [GR94]):

$$
\begin{aligned}
\int_{0}^{\infty} \int_{S^{n-1}} \frac{1}{\pi} \int_{0}^{\infty} \int_{S^{n-1}} F \mu(\alpha, \lambda) e^{-i \lambda r \alpha \cdot \beta} d \lambda d \alpha r^{m+2 l} Y_{m}(\beta) e^{-\epsilon r} d \beta r^{n-1} d r \\
=\frac{(2 \pi)^{n / 2}(-i)^{m}}{\pi} \int_{0}^{\infty} \int_{0}^{\infty} \tilde{\mu}_{Y_{m}}(\lambda) \frac{J_{m+(n-2) / 2}(\lambda r)}{(\lambda r)^{(n-2) / 2}} r^{m+2 l+n-1} e^{-\epsilon r} d r d \lambda \\
=\frac{(2 \pi)^{n / 2}(-i)^{m}}{\pi} \int_{0}^{\infty} \frac{\tilde{\mu}_{Y_{m}}(\lambda)}{\lambda^{(n-2) / 2}} \\
\quad \times\left(\frac{\partial}{\partial \epsilon}\right)^{2 l}\left[\int_{0}^{\infty} r^{m+(n / 2)} J_{m+(n / 2)-1}(\lambda r) e^{-\epsilon r} d r\right] d \lambda \\
=\frac{2^{m+(n / 2)}(2 \pi)^{n / 2}(-i)^{m}}{\pi^{3 / 2}} \Gamma(m+0.5(n+1)) \\
\quad \times\left(\frac{\partial}{\partial \epsilon}\right)^{2 l} \int_{0}^{\infty} \frac{\epsilon \lambda^{m}}{\left(\epsilon^{2}+\lambda^{2}\right)^{m+0.5(n+1)}} \tilde{\mu}_{Y_{m}}(\lambda) d \lambda \\
=\frac{2^{m+(n / 2)}(2 \pi)^{n / 2}(-i)^{m}}{\pi^{3 / 2}} \Gamma(m+0.5(n+1)) \\
\quad \times\left(\frac{\partial}{\partial \epsilon}\right)^{2 l}\left[\epsilon^{-(m+n)} \int_{0}^{\infty} \frac{(\lambda / \epsilon)^{m}}{\left(1+(\lambda / \epsilon)^{2}\right)^{m+0.5(n+1)}} \tilde{\mu}_{Y_{m}}(\lambda) d \lambda\right]
\end{aligned}
$$


where $J_{p}(z)$ is the Bessel function of the first kind, and

$$
\tilde{\mu}_{Y_{m}}(\lambda)=\int_{S^{n-1}} F \mu(\alpha, \lambda) Y_{m}(\alpha) d \alpha .
$$

Denote

$$
\Phi_{m}(t)=\frac{t^{m}}{\left(1+t^{2}\right)^{m+0.5(n+1)}} .
$$

Obviously,

$$
\Phi_{m}(t) \sim t^{-(m+n+1)} \sum_{k \geq 0} \frac{c_{m k}}{t^{2 k}}, t \rightarrow \infty,
$$

for some constants $c_{m k}$. Using (3.8, 3.10), 3.11) the results of BH86, (cf. Lemmas 4.3.3, 4.3.6, pp. 117-122, exercise 4.16, p. 201), and the results of [Won89] (Lemmas 1, 2, 6 in Chapter III) we obtain the asymptotic expansion of the last integral in (3.8) as $1 / \epsilon \rightarrow \infty$ :

$$
\begin{aligned}
\int_{0}^{\infty} & \Phi_{m}\left(\frac{1}{\epsilon} \lambda\right) \tilde{\mu}_{Y_{m}}(\lambda) d \lambda \sim \sum_{j=0}^{m+n-1} \epsilon^{j+1} M\left[\Phi_{m}, j+1\right] \frac{\tilde{\mu}_{Y_{m}}^{(j)}(0)}{j !} \\
& +\sum_{\substack{j>m+n \\
j-(m+n) \text { odd }}} \epsilon^{j+1} M\left[\Phi_{m}, j+1\right] \frac{\tilde{\mu}_{Y_{m}}^{(j)}(0)}{j !} \\
& +\sum_{\substack{j \geq m+n \\
j-(m+n) \text { even }}} \sum_{k=0}^{1} b_{k j} \epsilon^{j+1} \log ^{k} \epsilon, \epsilon \rightarrow 0^{+},
\end{aligned}
$$

where $b_{k, j}$ are some constants and $M\left[\Phi_{m}, z\right]$ denotes the (analytically continued) Mellin transform of $\Phi_{m}$ evaluated at $z$ (cf. BH86], pp. 110-116). The logarithmic terms appear in (3.12) because the poles of $M\left[\Phi_{m}, z\right]$ coincide in the right half-plane $\operatorname{Re} z \geq 1$ with some of the poles of $M\left[\tilde{\mu}_{Y_{m}}, 1-z\right]$. Since the order of differentiation with respect to $\epsilon$ in (3.8) is even, we see that the third sum in (3.12) does not contribute to the finite part of the limit in (3.7) and we get

$$
\begin{aligned}
\text { F.p. } \lim _{\epsilon \rightarrow 0^{+}} & \left(\frac{\partial}{\partial \epsilon}\right)^{2 l}\left[\epsilon^{-(m+n)} \int_{0}^{\infty} \Phi_{m}\left(\frac{1}{\epsilon} \lambda\right) \tilde{\mu}_{Y_{m}}(\lambda) d \lambda\right] \\
= & (2 l) ! M\left[\Phi_{m}, m+n+2 l\right] \frac{\tilde{\mu}_{Y_{m}}^{(m+n-1+2 l)}(0)}{(m+n-1+2 l) !} .
\end{aligned}
$$

Since $F \mu(\alpha, \lambda)$ is even, (3.9) implies that $\tilde{\mu}_{Y_{m}}(-\lambda)=(-1)^{m} \tilde{\mu}_{Y_{m}}(\lambda)$. Therefore, if $n$ is even, $\tilde{\mu}_{Y_{m}}^{(m+n-1+2 l)}(0)=0$ because $m$ and $m+n-1+2 l$ are of different parity. This implies that all the generalized moments of $f=R^{*} \mu$ vanish. Thus, our argument proves that $R^{*} \mathcal{S}\left(Z_{n}\right) \subset \mathcal{Y}_{1}$ if $n$ is even.

Let us prove that $\mathcal{Y}_{1} \subset R^{*} \mathcal{S}\left(Z_{n}\right)$. Suppose, first, that $n$ is odd. Let $f$ be a function with the properties (1.7), (1.8). Since the first line in (3.5) implies that the coefficients $c_{k m}$ decay at most polynomially as $m \rightarrow \infty\left(c_{k m}=O\left(m^{k-0.5(n-2)}\right)\right)$ and $c_{k m} \neq 0$ except in the case described in the second line of (3.5), we conclude from (3.1) that given $f_{k}(\beta)$ we can uniquely find the functions $\tilde{\mu}_{k}^{\prime}(\alpha) \in C^{\infty}\left(S^{n-1}\right)$ 
such that

$$
\begin{aligned}
& f_{k}(\beta)=\frac{1}{\pi} \int_{S^{n-1}} \overline{\Psi_{k}(\alpha \cdot \beta)} \tilde{\mu}_{k}^{\prime}(\alpha) d \alpha, \tilde{\mu}_{k}^{\prime}(-\alpha)=(-1)^{k} \tilde{\mu}_{k}^{\prime}(\alpha), \\
& \int_{S^{n-1}} \tilde{\mu}_{k}^{\prime}(\alpha) Y_{m}(\alpha) d \alpha=0, m \leq k-(n-1) .
\end{aligned}
$$

Let $\mu^{\prime}(\alpha, p) \in \mathcal{S}\left(Z_{n}\right)$ be any even function such that $F \mu^{\prime}(\alpha, \lambda) \sim \sum_{k \geq 0} \tilde{\mu}_{k}^{\prime}(\alpha) \lambda^{k}$, $\lambda \rightarrow 0$ (such a function exists by the Borel theorem Hor83, p. 16). By construction, $\varphi:=f-R^{*} \mu^{\prime} \in \mathcal{S}\left(\mathbb{R}^{n}\right)$. Denoting $\mu^{\prime \prime}(\alpha, p)=F_{\lambda \rightarrow p}^{-1}\left(\gamma_{n} \lambda^{n-1} \mathcal{F} \varphi(\lambda \alpha)\right)$ and $\mu=$ $\mu^{\prime}+\mu^{\prime \prime}$, we see that $\mu$ is even, $\mu \in \mathcal{S}\left(Z_{n}\right)$, and $R^{*} \mu=f$.

Let $n$ be even. Let $\tilde{\mu}_{k}^{\prime}(\alpha) \in C^{\infty}\left(S^{n-1}\right)$ be the unique solutions to the first equation in (3.14) with the property $\tilde{\mu}_{k}^{\prime}(-\alpha)=(-1)^{k} \tilde{\mu}_{k}^{\prime}(\alpha)$, and let $\mu^{\prime}(\alpha, p) \in$ $\mathcal{S}\left(Z_{n}\right)$ be any even function such that $F \mu^{\prime}(\alpha, \lambda) \sim \sum_{k \geq 0} \tilde{\mu}_{k}^{\prime}(\alpha) \lambda^{k}, \lambda \rightarrow 0$. By construction, $\varphi:=f-R^{*} \mu^{\prime} \in \mathcal{S}\left(\mathbb{R}^{n}\right)$. By assumption, the generalized moments of $f$ vanish. According to the above argument, if $\mu^{\prime} \in \mathcal{S}\left(Z_{n}\right)$ and $\mu^{\prime}$ is even, then the generalized moments of $R^{*} \mu^{\prime}$ vanish. Therefore, all the (usual) moments of $\varphi=f-R^{*} \mu^{\prime}$ vanish, that is, $\mathcal{F} \varphi(\xi)$ has zero of infinite order at $\xi=0$. Denoting $\mu^{\prime \prime}(\alpha, p)=F_{\lambda \rightarrow p}^{-1}\left(\gamma_{n}|\lambda|^{n-1} \mathcal{F} \varphi(\lambda \alpha)\right)$, we see that $\mu^{\prime \prime}$ is even and in $\mathcal{S}\left(Z_{n}\right)$. Therefore, $\mu=\mu^{\prime}+\mu^{\prime \prime} \in \mathcal{S}\left(Z_{n}\right), \mu$ is even, and $R^{*} \mu=f$.

Let us now prove the second part of the theorem. Suppose $\mu(\alpha, p)=0,|p| \geq$ $A>0$. Similarly to (3.9), denote

$$
\mu_{Y_{m}}(p)=\int_{S^{n-1}} \mu(\alpha, p) Y_{m}(\alpha) d \alpha, f_{Y_{m}}(r)=\int_{S^{n-1}} f(r \beta) Y_{m}(\beta) d \beta, f=R^{*} \mu
$$

It is easily checked that

$$
R^{*}\left(\mu_{Y_{m}}(p) Y_{m}(\alpha)\right)=f_{Y_{m}}(r) Y_{m}(\beta) .
$$

Indeed, multiplying both sides of $f=R^{*} \mu$ by $Y_{m}$ and integrating over $S^{n-1}$, we find

$$
\begin{aligned}
f_{Y_{m}}(r) & =\int_{S^{n-1}} \int_{S^{n-1}} \mu(\alpha, r \alpha \cdot \beta) Y_{m}(\beta) d \alpha d \beta \\
& =\sum_{l \geq 0} \int_{S^{n-1}} \int_{S^{n-1}} \mu_{Y_{l}}(r \alpha \cdot \beta) Y_{l}(\alpha) Y_{m}(\beta) d \alpha d \beta \\
& =\int_{S^{n-1}} \int_{S^{n-1}} \mu_{Y_{m}}(r \alpha \cdot \beta) Y_{m}(\alpha) Y_{m}(\beta) d \alpha d \beta \\
& =\int_{S^{n-1}} R_{(\alpha, p) \rightarrow r \beta}^{*}\left(\mu_{Y_{m}}(p) Y_{m}(\alpha)\right) Y_{m}(\beta) d \beta .
\end{aligned}
$$

Since $\int_{S^{n-1}} R_{(\alpha, p) \rightarrow r \beta}^{*}\left(\mu_{Y_{m}}(p) Y_{m}(\alpha)\right) Y_{l}(\beta) d \beta=0, l \neq m$, this proves (3.16). Applying (3.1) to the pair of functions $\mu_{Y_{m}}(p) Y_{m}(\alpha)$ and $f_{Y_{m}}(r) Y_{m}(\beta)$ and using (3.16), we conclude that

$$
f_{Y_{m}}(r) \sim \sum_{k \geq 0} \frac{f_{k m}}{r^{k+1}}, f_{k m}=\int_{S^{n-1}} f_{k}(\beta) Y_{m}(\beta) d \beta .
$$

To show that the series in (3.18) converges to $f_{Y_{m}}(r)$ for $r>A$, let us obtain an equation that relates $f_{Y_{m}}$ and $\mu_{Y_{m}}$. By the Funk-Hecke theorem,

$$
f_{Y_{m}}(r)=c_{m} \int_{-1}^{1} \mu_{Y_{m}}(r t) C_{m}^{((n-2) / 2)}(t)\left(1-t^{2}\right)^{(n-3) / 2} d t, c_{m} \neq 0,
$$


where $C_{m}^{(p)}$ is the Gegenbauer polynomial. Since $\mu_{Y_{m}}(s)=0,|s| \geq A$, 3.19) implies

$$
f_{Y_{m}}(r)=\frac{c_{m}}{r} \int_{-A}^{A} \mu_{Y_{m}}(s) C_{m}^{((n-2) / 2)}(s / r)\left(1-(s / r)^{2}\right)^{(n-3) / 2} d s, r \geq A .
$$

Since the function $\left(1-z^{2}\right)^{(n-3) / 2}$ is analytic in the disk $|z|<1$ and $C_{m}^{((n-2) / 2)}$ is a polynomial, we immediately prove the desired result. (In fact, if $n \geq 3$ is odd, (3.20) implies that $f_{Y_{m}}(r), r>A$, is a polynomial in $1 / r$.) Therefore, $R^{*} C_{0}^{\infty}\left(Z_{n}\right) \subset \mathcal{Y}_{2}$.

Let us now prove the inclusion $\mathcal{Y}_{2} \subset R^{*} C_{0}^{\infty}\left(Z_{n}\right)$. Let $n$ be odd. Using the inversion formula $\left(R^{*}\right)^{-1} h=\gamma_{n} R(-\Delta)^{(n-1) / 2} h$ and substituting $h(r \beta)=f_{Y_{m}}(r) Y_{m}(\beta)$, we find

$$
\mu_{Y_{m}}(p) Y_{m}(\alpha)=\gamma_{n} R\left(-\Delta_{x}\right)^{(n-1) / 2}\left(f_{Y_{m}}(r) Y_{m}(\beta)\right), x=r \beta .
$$

Assumptions (1.7), (1.8), and (1.10), imply that $r f_{Y_{m}}(r), r>A$, is a polynomial in $1 / r$ that contains the powers $k \leq m+n-3, k+m$ is even. Applying powers of $\Delta_{x}$ to $Y_{m}(\beta) / r^{k+1}$ and using the identities

$$
\Delta_{x}=\frac{1}{r^{n-1}} \frac{\partial}{\partial r}\left(r^{n-1} \frac{\partial}{\partial r}\right)+\frac{1}{r^{2}} \Delta_{\alpha},\left(\Delta_{\alpha}+m(n-2+m)\right) Y_{m}(\alpha)=0,
$$

we get

$$
\begin{aligned}
& \Delta_{x} \frac{Y_{m}(\beta)}{r^{k+1}}=\frac{(k+m+1)(k-m-n+3)}{r^{2}} \frac{Y_{m}(\beta)}{r^{k+1}}, \\
& \Delta_{x}^{2} \frac{Y_{m}(\beta)}{r^{k+1}}=\frac{(k+m+3)(k-m-n+5)}{r^{2}} \Delta_{x} \frac{Y_{m}(\beta)}{r^{k+1}}, \ldots, \\
& \Delta_{x}^{(n-1) / 2} \frac{Y_{m}(\beta)}{r^{k+1}}=\frac{(k+m+n-2)(k-m)}{r^{2}} \Delta_{x}^{(n-3) / 2} \frac{Y_{m}(\beta)}{r^{k+1}} .
\end{aligned}
$$

This implies

$$
\Delta_{x}^{(n-1) / 2}\left(f_{Y_{m}}(r) Y_{m}(\beta)\right)=Y_{m}(\beta) \sum_{\substack{k \leq m-2 \\ k+m \text { even }}} \frac{c_{k}}{r^{k+n}}, r \geq A
$$

Taking the Radon transform similarly to (2.3) in [Kat97], we get $R \Delta_{x}^{(n-1) / 2}\left(f_{Y_{m}} Y_{m}\right)$ $=0,|p| \geq A$. Equation (3.21) implies $\mu_{Y_{m}}(p)=0,|p| \geq A$. Since $m \geq 0$ is arbitrary, $\mu(\alpha, p)=0,|p| \geq A$.

Let $n$ be even. We have

$$
F \mu_{Y_{m}}(\lambda) Y_{m}(\alpha)=\gamma_{n}|\lambda|^{n-1} \mathcal{F}_{r \beta \rightarrow \lambda \alpha}\left(f_{Y_{m}}(r) Y_{m}(\beta)\right)
$$

As before, denote $g_{l m}=\left(\partial / \partial x_{l}\right)^{n-1}\left(f_{Y_{m}} Y_{m}\right)$. Then

$$
F \mu_{Y_{m}}(\lambda) Y_{m}(\alpha)=(-1)^{n / 2} \frac{\gamma_{n}}{\alpha_{l}^{n-1}}(-i \operatorname{sgn} \lambda) \mathcal{F} g_{l m}(\lambda \alpha), \alpha_{l} \neq 0
$$

that is,

$$
\left(\mathcal{H}_{p \rightarrow t} \mu_{Y_{m}}(p)\right) Y_{m}(\alpha)=-(-1)^{n / 2} \frac{\gamma_{n}}{\alpha_{l}^{n-1}} R g_{l m}(\alpha, t), \alpha_{l} \neq 0 .
$$

By assumption, $f_{Y_{m}}(r)$ is the sum of its asymptotic series on the domain $r>A$. From (1.7) and (1.10)

$$
f_{Y_{m}}(r)=\sum_{\substack{k \geq 0 \\ k+m \text { even }}} \frac{c_{k}}{r^{k+1}}, r>A
$$


for some constants $c_{k}$. Since $n$ is even, repeated differentiation of $f_{Y_{m}}(r) Y_{m}(\beta)$, $x=r \beta$, with respect to $x_{l}$ gives

$$
g_{l m}(r \beta)=\sum_{j \leq m+n-1} Y_{j}(\beta) \sum_{\substack{k \geq 0 \\ k+j \text { odd }}} \frac{g_{k j l}}{r^{k+n}}, r>A,
$$

for some constants $g_{k m l}$, and for each $j$ the series with respect to $k$ is absolutely convergent. Substituting into the definition of the Radon transform, integrating, and using the Funk-Hecke theorem we obtain

$$
\begin{aligned}
& R g_{l m}(\alpha, t)=\sum_{j \leq m+n-1} Y_{j}(\alpha) \sum_{\substack{k \geq 0 \\
k+j \text { odd }}} \frac{\hat{g}_{k j l}}{t^{k+1}},|t|>A \\
& \hat{g}_{k j l}=g_{k j l} \frac{\left|S^{n-2}\right|}{C_{j}^{((n-2) / 2)}(1)} \int_{0}^{1} s^{k} C_{j}^{((n-2) / 2)}(s)\left(1-s^{2}\right)^{(n-3) / 2} d s .
\end{aligned}
$$

The formula for $R g_{l m}$ is first derived for $t>A$, and then extended to $t<-A$ using that $R g_{l m}$ is even and $\hat{g}_{k j l} \neq 0$ only when $k+j$ is odd. Thus, $R g_{l m}(\alpha, t)$ is the sum of its asymptotic series on the domain $|t|>A$ and, therefore, $R g_{l m}(\alpha, t)$ can be extended (in the $t$-variable) as an analytic function to the domain $|z|>A, z \in \mathbb{C}$. Using the inversion formula for the Hilbert transform, we have

$$
\begin{aligned}
\mu_{Y_{m}}(p) Y_{m}(\alpha) & =\frac{\gamma_{n}(-1)^{n / 2}}{\alpha_{l}^{n-1}} \frac{i}{2} \lim _{\epsilon \rightarrow 0^{+}}\left[R g_{l m}(\alpha, p+i \epsilon)-R g_{l m}(\alpha, p-i \epsilon)\right]=0 \\
\alpha_{l} & \neq 0,|p|>A
\end{aligned}
$$

Since $l$ is arbitrary, we finish the proof. The theorem is proved.

Remark 1. The following observation appears to be of interest. Let $\mu \in \mathcal{S}\left(Z_{n}\right)$ and $f=R^{*} \mu$. Clearly, $F \mu(\alpha, \lambda) \sim \sum_{k=0}^{\infty} \tilde{\mu}_{k}(\alpha) \lambda^{k}, \lambda \rightarrow 0$, where $\tilde{\mu}_{k}(\alpha)=\left(i^{k} / k !\right)$ $\times \int_{-\infty}^{\infty} p^{k} \mu(\alpha, p) d p$. If $n$ is even, none of the zero eigenvalues $c_{k m}$ of the integral operator in (3.1) fall onto nonzero harmonics of $\tilde{\mu}_{k}(\alpha)$, that is, $\tilde{\mu}_{k}(\alpha)$ can be uniquely determined knowing $f_{k}(\beta)$ by solving (3.1). However, if $n$ is odd, some of the $c_{k m}$ 's that correspond to nonzero harmonics of $\tilde{\mu}_{k}(\alpha)$ are zero. Hence, unique recovery of $\tilde{\mu}_{k}(\alpha)$ from (3.1) is not possible. It turns out that determination of the 'lost' harmonics is still possible without full inversion of $f$. This can be done by using the generalized moments. Indeed, from (3.9)

$$
\int_{S^{n-1}} \tilde{\mu}_{k}(\alpha) Y_{m}(\alpha) d \alpha=\frac{1}{k !} \tilde{\mu}_{Y_{m}}^{(k)}(0) .
$$

Equations (3.7)-(3.13) imply that $M_{P}, P=r^{m+2 l} Y_{m}$, and $\tilde{\mu}_{Y_{m}}^{(k)}(0), k=m+n-1+$ $2 l, l \geq 0$, are proportional. Comparing (3.32) and (3.5) we see that these values of $k$ correspond precisely to the 'lost' components. By (3.10) and (3.251.11) in [GR94,

$$
M\left[\Phi_{m}, m+n+2 l\right]=0.5 B(m+l+(n / 2), 0.5-l) \neq 0,
$$

where $B$ is the beta-function. Hence, by $(3.13) \tilde{\mu}_{Y_{m}}^{(m+n-1+2 l)}(0)$ and the 'lost' components can be found knowing $M_{P}, P=r^{m+2 l} Y_{m}$. 


\section{REFERENCES}

[BH86] N. Bleistein and R. Handelsman, Asymptotic expansions of integrals, Dover, New York, 1986. MR 89h:41049

[Fed77] M. V. Fedoriuk, Metod perevala, Nauka, Moscow, 1977, (Russian). MR 58:22580

[GGV66] I.M. Gelfand, M.I. Graev, and N.Ya. Vilenkin, Generalized functions. Volume 5: Integral geometry and representation theory, Academic Press, New York, 1966. MR 55:8786e; MR 34:7726

[Gon84] F. B. Gonzalez, Radon transforms on Grassmann manifolds, Ph.D. thesis, M.I.T., 1984.

[Gon87] F. B. Gonzalez, Radon transforms on Grassmann manifolds, J. Funct. Anal. 71 (1987), 339-362. MR 89a:53081

[GR94] I. S. Gradshteyn and I. M. Ryzhik, Table of integrals, series, and products, 5th ed., Academic Press, Boston, 1994. MR 94g:00008

[GS64] I. M. Gelfand and G.E. Shilov, Generalized functions. Volume 1: Properties and operations, Academic Press, New York, 1964. MR 55:8786a

[Hel65] S. Helgason, The Radon transform on Euclidean spaces, compact two-point homogeneous spaces and Grassmann manifolds, Acta Mathematica 113 (1965), 153-170. MR 30:2530

[Hel80] S. Helgason, The Radon transform, Birkhäuser, Boston, 1980. MR 83f:43012

[Hel82] S. Helgason, Ranges of Radon transforms, Proceedings of Symposia in Applied Mathematics, Vol. 27 (Providence, RI) (L. Shepp, ed.), Amer. Math. Soc., 1982, pp. 63-70. MR 84h:44016

[Her83] A. Hertle, Continuity of the Radon transform and its inverse on Euclidean spaces, Math. Z. 184 (1983), 165-192. MR 86e:44004a

[Her84] A. Hertle, On the range of the Radon transform and its dual, Math. Ann. 267 (1984), 91-99. MR 86e:44004b

[Hor83] L. Hormander, The analysis of linear partial differential operators, Vol. I, SpringerVerlag, New York, 1983. MR 85g:35002a

[Kat97] A. Katsevich, Range of the Radon transform on functions which do not decay fast at infinity, SIAM Journal of Mathematical Analysis 28 (1997), no. 4, 852-866. MR 98g:44001

[Lou84] A. K. Louis, Orthogonal function series expansions and the null space of the Radon transform, SIAM J. Math. Anal. 15 (1984), 621-633. MR 85j:44003

[LP70] P. Lax and R. Phillips, The Paley-Wiener theorem for the Radon transform, Comm. Pure Appl. Math. 23 (1970), 409-424. MR 42:8189

[Lud60] D. Ludwig, The Radon transform on Euclidean spaces, Comm. Pure Appl. Math. 19 (1960), 49-81. MR 32:8064

[Ram95] A.G. Ramm, The Radon transform is an isomorphism between $L^{2}(B)$ and $H_{e}\left(Z_{a}\right)$, Appl. Math. Lett. 8 (1995), 25-29. CMP 96:02

[Ram96] A.G. Ramm, Inversion formula and singularities of the solution for the back-projection operator in tomography, Proc. Amer. Math. Soc. 124 (1996), 567-577. MR 96d:44001

[RK96] A. Ramm and A. Katsevich, The Radon transform and local tomography, CRC Press, Boca Raton, Florida, 1996. MR 97g:44009

[SM88] D. C. Solmon and W. Madych, A range theorem for the Radon transform, Proceedings of the Amer. Math. Soc. 104 (1988), 79-85. MR 90i:44003

[Sol87] D.C. Solmon, Asymptotic formulas for the dual Radon transform and applications, Math. Z. 195 (1987), 321-343. MR 88i:44006

[SSW77] K. Smith, D. Solmon, and S. Wagner, Practical and mathematical aspects of the problem of reconstructing objects from radiographs, Bull of Amer. Math. Soc. 83 (1977), 12271270. MR 58:9394a; MR 58:9394b

[Won89] R. Wong, Asymptotic approximations of integrals, Academic Press, Boston, 1989. MR 90j:41061

Department of Mathematics, University of Central Florida, Orlando, Florida 32816

E-mail address: akatsevi@pegasus.cc.ucf.edu 